Bożena Zwolińska

Akademia Górniczo-Hutnicza im. Stanisława Staszica w Krakowie

E-mail: bzwol@agh.edu.pl

Małgorzata Kowalczyk

Akademia Górniczo-Hutnicza im. Stanisława Staszica w Krakowie

E-mail: mkowalczyk@agh.edu.pl

Paweł Gara

Akademia Górniczo-Hutnicza im. Stanisława Staszica w Krakowie

E-mail: pgara@agh.edu.pl

\title{
LEAD TIMES OF CRITICAL PATH TASKS IN CONVERGENT PRODUCTION
}

\begin{abstract}
Introduction: Now, in time of Industry 4.0, the development trend in many companies is integration of the real world and the virtual world. In accordance with the Industry 4.0 assumptions, the factories are fully automated and allow exchange of any information during production. Along with digitization of all areas of production company and development of numerical machines, implementation of computationally complex production control support algorithms becomes possible. One of the company growth aspects, in line with the Industry 4.0 mainstream, is product customization. Depending on the specific character of industry, there are different methods to match the product to individual expectations of the customer.
\end{abstract}

Materials and results: The chapter includes a study of a real production facility that offers a wide range of products and at the same time is characterized by a high degree of product match to the individual expectations of customers. The result is a relatively high dynamics of labour intensity levels of individual production structure areas. In addition, the offered range is a complex system consisting of many semi-finished products (which also are complex systems) and its final form offered to the customer is made during the so-called convergent production. Taking into account the multifaceted complexity of a production system, including the complexity of material flow stream, it becomes extremely important to relatively accurately evaluate the order lead times in order to keep a high customer service KPI (Key Performance Indicators). 
The chapter presents an algorithm for production tasks lead times that accounts for characteristic features of the studied production system. The algorithm is a modification of the PERT (Project Evaluation and Review Technique) method in which the evaluation of the cumulated production tasks lead time for critical path is more accurate than in the classical PERT method.

The chapter presents comparative results of two evaluation methods of production order lead time: one according to the classical PERT method and the other according to the proposed algorithm. The analyses were carried out for actual data collected on selected few days in one month. The data selection criteria included varied labour intensity levels of individual production areas which are directly determined by varied customer orders.

The comparative analyses of two methods of critical task lead times evaluation refer to three representative product groups. Selection criterion of product sets from the cumulated demand number during a year and a demand variability index. The presented analyses do not include all possible cases of work labour intensity variation during production which will be accounted for during further research.

Conclusions: The chapter presents an algorithm for determination of cumulated production tasks lead times based on a modified classical PERT method. The proposed algorithm accounts for operating parameters of the studied system and an improvement of lead time probability estimation in individual planning itineraries of individual streams of processed materials. The proposed algorithm should be subjected to a detailed validation for longer time perspectives, e.g. one year. The reason is a seasonal character of demand at the turn of the year (from November to February). In addition, the next algorithm verification tests should account for the holistic approach of the entire production system and the multitude of critical paths for various product groups. The obtained results will form a basis to develop a tasks ranging model for the studied class of production systems.

Keywords: PERT, critical path, convergent production

\section{INTRODUCTION}

The graph theory is a division of mathematics and computer science which allows solving many practical problems with the use of graphs (diagrams of relations and interconnections). The mathematical models developed in the graph theory account for the variables which significantly affect the studied process (project). In production structures, the graph theory models which are used most often include CPM - Critical Path Method and

\section{6}

Brzeziński Ł. et al. (Eds.), Modern solutions in production and warehousing, Spatium, Radom 2019. 
PERT Project Evaluation and Review Technique. Both CPM and PERT are used to plan and control the project which is a set of interdependent tasks. The critical path is established in both methods and determines the project completion time [Idźkiewicz 1967]. The critical path is used to determine the earliest and latest dates for individual events and the available time reserve (slack) for individual stages. The basic difference between CPM and PERT lies in the deterministic or probabilistic analyses of individual events. In the CPM, the times of individual processes are considered as static values, clearly determined for a given $\Delta t$. In the PERT, the time of a single event is the expected value of random variable conforming to the PERT distribution. Based on this, the whole project which is a set of many interdependent tasks, has a probabilistic character [Nafkha 2016]. Therefore, the probability values for completion of the whole project are determined at successive stages of the PERT analysis.

There are many simplifying assumptions in a traditional PERT analysis which are however sufficiently accurate in the majority of cases. In practice, there are areas of business where the PERT analysis should be used because of its advantages, but the classical method is not sufficiently accurate. Therefore, the literature on the PERT includes many solutions to improve the method's weaknesses. There are two basic groups: 1) evaluate the variance or standard deviation of individual tasks more accurately; and 2) evaluate the probability in individual nodes more accurately [Kamburowski 1997]. In the first group, the more accurate results are obtained based on the quantile evaluation. There are three cases here: evaluation based on three, four or six quantiles [Milian 2006]. In the second group, the more accurate results are obtained based on the better fit of the probability distribution of random variable determining the task duration in a node, e.g. by applying the beta-PERT distribution or the Pearson distribution. Each attempt to improve the PERT accuracy relative to its classical approach is connected with the degree of calculations complexity. This in turn makes the improved method unsuitable for cases where the precise accuracy is not necessary. The research on improving the PERT evaluation accuracy is continuing, with the use of many probability theory aspects, inter alia probability distribution curve, distribution kurtosis (socalled distribution flatness ratio), distribution skewness, etc. [Fatemi Ghomi and Rabbani 2003 ; Mazlum and Guneri 2010].

The chapter intends to develop an algorithm for determining the cumulative project duration according to the PERT method taking into account various skewness values of individual tasks. The developed algorithm is an attempt to modify the classical PERT 
method which scales the expected value of a single task using the skewness contribution parameter. The skewness contribution parameter is determined based on the percent share of skewness of an individual distribution relative to all skewness values of the critical path. The proposed method does not increase significantly the level of complication and calculation complexity relative to the classical PERT method, but is its more accurate alternative.

\section{CASE STUDY DESCRIPTION}

The majority of publications on analyses, attempts to optimize or improve the production processes, concerns the series or large-series production. In almost all mass production cases it is possible to use the mean value as a sufficient reality description parameter.

The Gauss distribution is one of the most often used random variable distributions of the reality description. According to the central limit theorem (CTL), the random variables describing the value of the estimated parameter, which are determined by an infinite number of independent random events of identical distributions and finite variances, have the normal distribution. Two conditions must be met in order to use the Gauss distribution to describe a feature: random variables defined by any identical distribution must be independent, and - at the same time - the number of their possible occurrences must be infinite. The approximation with the Gauss distribution is more accurate when the empirical sample size is greater.

The chapter considers a system with a wide diversity of the product range and of components making up a single final product. These fact determine that products are made in short batches in the make-to-order (MTO) system. In such case, defining the task times on individual machines according to the normal distribution is burdened with an unacceptable error. Therefore, the authors have used another probability distribution to describe the production task times.

The main need for the modification of cumulative value of production times determined in accordance with the PERT method has resulted from the estimation errors of purchase orders' lead times. The studied production system is agile and flexible. A quick adaptation of the company to customers' orders which vary in terms of products and sizes determines the dynamics of operating states changes in the manufacturing system. In addition, the studied system makes the offered product range in short batches consisting of several identical final products. The analysis of historical data has indicated that during a single settlement period of any length, the maximum order size was 7 pieces of identical final products. The demand variability is shown in the boxplot - Fig. 1 .

\section{8}

Brzeziński Ł. et al. (Eds.), Modern solutions in production and warehousing, Spatium, Radom 2019. https://doi.org/10.17270/B.M.978-83-66017-87-0.11 


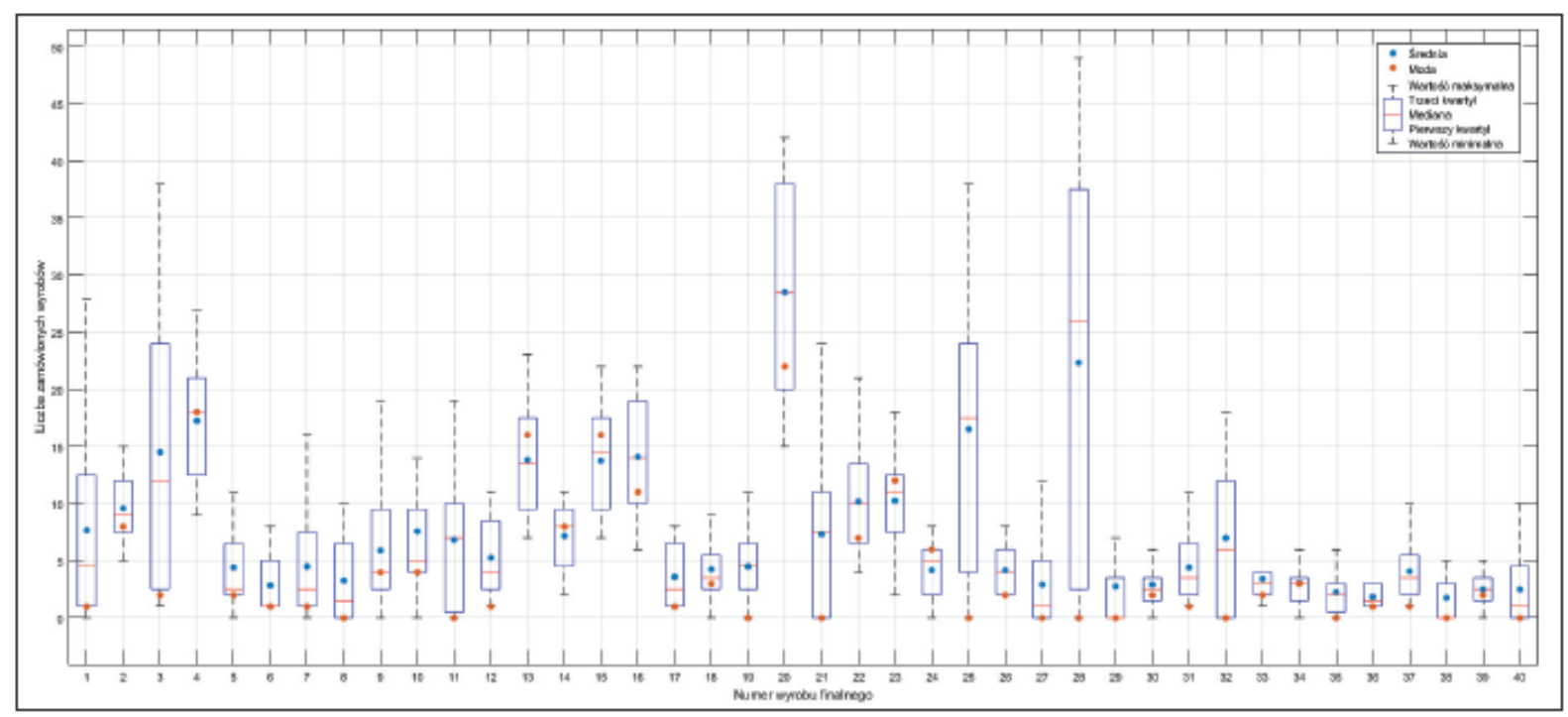

Fig. 1 Boxplot - demand variability in the studied production system. Source: own work

A relatively high demand variability in terms of order size, as well as product range diversity determines a huge variation of operation states in the studied production system. In addition, depending on $\Delta t$ for a single technical facility, there are different mean task duration times. Such multi-aspect variation makes a deterministic system behave randomly in a longer time frame. Thus, frequent estimation errors occurred during the determination of the time necessary to manufacture a precisely defined set of final products; such errors were unacceptable at this scale of production volume.

\section{PERT DISTRIBUTION AND PERT METHOD}

The PERT distribution belongs to the family of continuous probability distributions and is a transformation of the beta distribution. The transformation involves rescaling the random variable carrier from interval $[0,1]$ to interval $[a, c]$ when $a<c$ [Devore 2012]. Value $a$ is the minimum and $c$ is the maximum of the random variable distribution. Consequently, the PERT distribution is defined by three parameters: $a$ - minimum, $b$ - mode, $c$ - maximum. Consequently, the PERT distribution is defined by three parameters: $a$-minimum, $b$ - mode, $c$-maximum. Hence:

$$
E t=\frac{a+4 b+c}{2+k}
$$

where: $E t$ - expected value of random variable $t$ conforming to the PERT distribution, $a$ - minimum distribution value, $c$-maximum distribution value, $b$ - modal distribution value of random variable $t, k$-modal value multiplicity (for PERT always $k=4$ ). 
There are three often used methods of matching (estimation) of distribution parameters: $a, b, \mathrm{c}$ and $\mathrm{k}$ for a simple sample (e.g. set of production tasks completion times determined by timekeeping: 1) reading the values directly from the dataset, i.e. $a=\min , c=\max$ etc.; 2) determining the parameters $a, b, c$ and $k$ using the method of moments; or 3) determining the parameters $a, b, c$ and $k$ using the maximum likelihood method. Various estimation methods for distribution parameters are used depending on the required degree of accuracy and available computing power.

Assumptions for the PERT distribution:

- relationship between the distribution parameters: $0<a<b<c$;

- modal value multiplicity: $k=4$ (approximation of empirical data with the PERT distribution where $k>4$ is allowed, however the beta-PERT distribution gives a more accurate estimation);

- $\quad$ skewness from the empirical sample must be in the range * :

$$
A_{S} \in\left[-\sqrt{\frac{7}{5}}, \sqrt{\frac{7}{5}}\right]
$$

* determined skewness interval limits result from the distribution properties;

- kurtosis from the empirical sample $K_{S}>0$;

- number of manufactured semi-finished products $n=[30]-$ it is possible to use a distribution for the sample size greater than 30 (provided that the mode multiplicity is 4 ), however, due to the calculation complexity, in practice e.g. a Gauss distribution is used when the conditions for its application are met [Zwolińska 2019].

The PERT distribution is an alternative to the triangular distribution because it puts less emphasis on the extreme values (minimum and maximum) by smoothing the tails shapes. Figure 2 presents a comparison of the PERT and triangular distributions. 


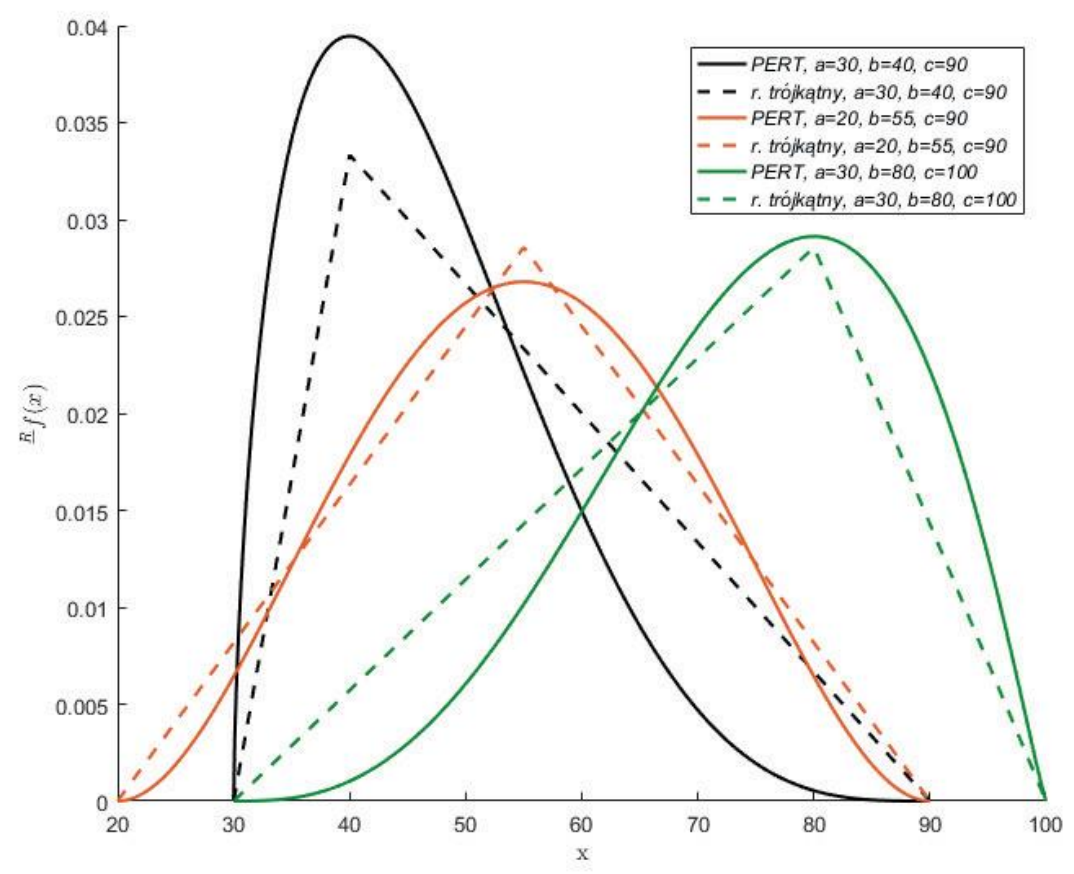

Fig. 2. Comparison of triangular and PERT distributions. Source: own work.

Note however that the PERT distribution is more accurate when the multiplicity of the most probable value equals 4 . This is a significant simplification, however usually considered sufficiently accurate. A modification of the PERT distribution with any modal value multiplicity greater than four has been applied in the beta-PERT distribution, allowing the tasks durations and project completion time to be determined with a higher accuracy. However, due to the level of difficulty and complex calculations, the beta-PERT distribution is not widely used.

The PERT method involves the time estimation according to the expected value of the PERT distribution as per the formula (1). The variance is determined in accordance with the formula:

$$
D^{2}(t)=\left(\frac{c-a}{6}\right)^{2}
$$

where: $D^{2}(t)$ - variance of a single random variable $t ; a$ and $c$-estimated minimum and maximum values of the PERT distribution, respectively.

Knowing that the times of individual production tasks are independent of one another, the standard deviation of cumulative production times will be calculated according to the classical PERT method. Hence: 


$$
D T=\sqrt{\sum_{i=1}^{n}\left(\frac{c_{i}-a_{i}}{6}\right)^{2}}
$$

where: $D T$ - standard deviation of random variable $T ; T$ - random variable defining the cumulative duration of all tasks on the critical path; $a_{i}$ and $c_{i}$ - estimated minimum and maximum values of PERT distribution of a single random variable $t_{i}$ for $i=1,2, \ldots, n$, where $n$ is the maximum number of tasks on the critical path.

Using the linearity property of the expected value of independent random variables $t_{i}$ for $i=1,2, \ldots, n$, the $E T$ can be calculated according to the formula:

$$
E T=\sum_{i=1}^{n} E\left(t_{i}\right)
$$

where: $E T$ - expected value of random variable $T$, which defines the cumulative value of the most probable duration of production tasks on the critical path ( $E T$ defines the lead time of an order or production, depending on the assumptions).

\section{ALGORITHM FOR ET DETERMINATION ACCOUNTING FOR $A_{S}$}

In majority of cases, the histograms of production tasks durations are right-skewed distributions. In such distributions, a significant portion of the probability mass is concentrated on the left-hand side of the plot. The classical PERT method does not account for this aspect. Hence, the proposition to develop an algorithm for estimating the cumulative value of the expected duration of the critical path tasks which will include the influences of the existing skewness values. The developed algorithm consists of five calculation steps. Before the calculations, the data on production tasks durations are collected by means of timekeeping. Hence, for the resultant simple sample of independent random variables $t_{i}$, for $i=1,2, \ldots, n$, where: $t_{i}$ - random variable defining the task duration on the $i$ th production facility; $n$ - number of facilities performing the production processes on the critical path. Then:

$$
\forall i=1,2, \ldots, n \quad t_{i} \sim F_{i}
$$

where each random variable $t_{i}$ is defined by the distribution of the cumulative distribution function $F_{i}$.

The approximation of production times is correct if $\forall i=1,2, \ldots, n$ the following conditions are met: 
- the distribution carrier is limited with the values: minimum $-a_{i}$, maximum $-c_{i}$; in addition $a_{i}<c_{i}$;

- for the simple sample of distribution $t_{i}$, the mode multiplicity $b_{i}=4$ and $a_{i}<b_{i}<c_{i}$.

When these conditions are met, $\forall i=1,2, \ldots, n$ the random variable $t_{i}$ can be approximated using the PERT distributions with parameters $a_{i}, b_{i}, c_{i}$.

The calculation methodology according to the proposed algorithm (five successive steps) is presented below.

Step 1 - determine the expected value $E\left(t_{i}\right)$ of variable $t_{i}$ defining the task duration on a single facility (machine).

$$
\begin{aligned}
& \forall i=1,2, \ldots, n: \\
& E\left(t_{i}\right)=\frac{a_{i}+4 b_{i}+c_{i}}{6}
\end{aligned}
$$

where: $E\left(t_{i}\right)$ - expected value of random variable $t_{i}, a_{i}, b_{i}, c_{i}$ - respectively: minimum, modal and maximum values of random variable $t_{i}$.

Step 2 - determine the skewness of random variable $t_{i}$

$$
\forall i=1,2, \ldots, n \text { : }
$$

$$
A_{S}\left(t_{i}\right)=\frac{2\left(\beta_{i}-\alpha_{i}\right) \sqrt{\alpha_{i}+\beta_{i}+1}}{\left(\alpha_{i}+\beta_{i}+2\right) \sqrt{\alpha_{i}+\beta_{i}}}
$$

where: $A_{S}\left(t_{i}\right)$-skewness of random variable $t_{i} ; \alpha_{i}, \beta_{i}$ - shape and scale parameters of the probability density function of random variable $t_{i}$ determined according to the formulas:

$$
\alpha_{i}=1+4 \frac{b_{i}-a_{i}}{c_{i}-a_{i}}
$$

and

$$
\beta_{i}=1+4 \frac{c_{i}-b_{i}}{c_{i}-a_{i}}
$$

Step 3 - determine the weights $W_{A_{S}\left(t_{i}\right)}$ of proportional skewness contribution $A_{S}\left(t_{i}\right)$ $\forall i=1,2, \ldots, n$ such that $A_{S}\left(t_{i}\right) \neq 0$ determined is the weight $W_{A_{S}\left(t_{i}\right)}$ of skewness $A_{S}\left(t_{i}\right)$ according to the formula: 


$$
W_{A_{S}\left(t_{i}\right)}=\frac{\left|A_{S}\left(t_{i}\right)\right|}{\sum_{i=1}^{n}\left|A_{S}\left(t_{i}\right)\right|}
$$

where: $W_{A_{S}\left(t_{i}\right)}$ - weight of skewness contribution for random variable $t_{i} ; A_{S}\left(t_{i}\right)$ - skewness of variable $t_{i}$.

The weights $W_{A_{S}\left(t_{i}\right)}$ are determined only for non-zero $A_{S}\left(t_{i}\right)$, i.e. the distribution of random variable $t_{i}$ is asymmetrical (left- or right-skewed).

Step 4 - determine the parameter $P_{A_{S}\left(t_{i}\right)}$ scaling the contribution of expected value $E\left(t_{i}\right)$ $\forall i=1,2, \ldots, n$ such that $A_{S}\left(t_{i}\right) \neq 0$ determined is the scaling parameter $P_{A_{S}\left(t_{i}\right)}$ of expected value $E\left(t_{i}\right)$ according to the formula:

$$
P_{A_{S}\left(t_{i}\right)}=\frac{W_{A_{S}\left(t_{i}\right)} \cdot 1}{\frac{1}{m}}=W_{A_{S}\left(t_{i}\right)} \cdot m
$$

where: $m=n-l$ for $l$ defining the number of random variables whose $A_{S}\left(t_{i}\right)=0 ; P_{A_{S}\left(t_{i}\right)}$ - scaling parameter for contribution of expected value $E\left(t_{i}\right)$.

If for a facility $i$, skewness $A_{S}\left(t_{i}\right)=0$, then $P_{A_{S}\left(t_{i}\right)}=1$

Step 5 - determine the cumulative expected value ET for the critical path.

In the classical PERT method, for the determination of the cumulative duration of tasks on the critical path - formula (5), it was assumed that the number of individual $t_{i}$ is so great that the central limit theorem of the sum of mean production tasks times can be used. This assumption was used in the algorithm proposed by the authors, and in addition the skewness of individual variables $t_{i}$ was accounted for. In formula (5), the parameters scaling the expected value $E\left(t_{i}\right)$ are equal to one (that is $P_{A_{S}\left(t_{i}\right)}=1$ ). In the proposed method, the cumulative expected value of the critical path is determined according to:

$$
E T=\sum_{i=1}^{n} P_{A_{s}\left(t_{i}\right)} \cdot E\left(t_{i}\right)
$$

where: $E T$ - cumulative value of the critical path; $P_{A_{S}\left(t_{i}\right)}-$ scaling parameter for contribution of expected valu $E\left(t_{i}\right)$.

The classical PERT method is based on assumptions that are not always met. The knowledge of exact distribution of task times in the network of events allows avoiding many errors and estimating the task times with a better accuracy. The application of more accurate 
PERT methods causes some calculation difficulties resulting from the relationships between random variables.

\section{CONCLUSIVE REMARKS}

The chapter presents an algorithm for determination of the expected value of cumulative production task times based on the modified classical PERT method. The proposed algorithm is used to determine ET taking into account the scaling parameters $P_{A_{S}\left(t_{i}\right)}$ of empirical skewness of individual random variables $t_{i}$. The scaling parameters $P_{A_{s}\left(t_{i}\right)}$ were determined taking into account $W_{A_{S}\left(t_{i}\right)}$, that is the weight contribution of skewness $A_{S}\left(t_{i}\right)$ of individual $t_{i} \sim \operatorname{PERT}\left(a_{i}, b_{i}, c_{i}\right)$.

The purpose of the developed algorithm is to improve the estimation of the expected critical path lead time. Please note that the time estimate obtained with the use of PERT method is correct when the network contains only one dominating critical path. When the dominating critical path is not there, the task completion times are underestimated. The proposed algorithm should be subject to a detailed validation in order to verify its effectiveness in practice. The verification of correctness should account for the demand seasonality and variability as well as for the product range variations that occur in the studied class of production systems. The obtained results should form a basis to develop a tasks scheduling model for the studied class of convergent production systems.

\section{REFERENCES}

Devore L. J., 2012, Probability and Statistics for Engineering and the Sciences, Books/Cole, Boston.

Fatemi Ghomi S.M.T., Rabbani M., 2003, A new structural mechanism for reducibility of stochastic PERT networks, European Journal of Operational Research, vol. 145, issue 2, s. 394-402.

Idźkiewicz A., 1967, PERT Metody analizy sieciowej, Państwowe Wydawnictwo Naukowe, Warszawa.

Kamburowski J., 1997, New validations of PERT times, Omega, International Journal of Management Science, nr 25, s. 323-328. 
Mazlum M., Guneri, A.F., 2010 CPM, PERT and Project Management with Fuzzy Logic Technique and Implementation on a Business, Procedia - Social and Behavioral Sciences, vol. 210, s. 348-357.

Milian Z., 2006, Metody określania rozkładu czasu w realizacji przedsięwzięć budowlanych w acyklicznych sieciach stochastycznych, Wydawnictwo Politechniki Krakowskiej, Kraków.

Nafkha R., 2016, The PERT method in estimating project duration, Information Systems in Management, vol. 5, nr 4, s. 542-550.

Zwolińska B., 2019 Modelowanie procesów konwergentnych w złożonych systemach produkcyjnych, Wydawnictwa AGH, Kraków. 